\title{
Implementation of the Masquelet Technique in Complicated Septic Non-union of the Ulna-A Case Report
}

\author{
Christos K. Kyriakopoulos ${ }^{1}$, Ioannis K. Trintafyllopoulos ${ }^{2}$, Thomas A. Kostakos ${ }^{3}$ and Athanasios T. Kostakos ${ }^{1}$ \\ 1. ST' Orthopaedic Department, General Hospital of Attica 'KAT', Athens, Greece \\ 2. Laboratory for the Research of Musculoskeletal System, Medical School, National and Kapodistrian University of Athens, Greece \\ 3. General Surgery Department, General Hospital Paidon-Pedelis, Athens, Greece
}

\begin{abstract}
Long bone septic non-union by MRSA (methicillin-resistant Staphylococcus aureus) is always a challenge for the orthopaedic society. Traditional treatment options include distraction osteogenesis methods and vascularised bone grafting. These techniques require a high level of expertise and are frequently involved with a variety of side effects or complications. We present a rare case of ulnar fracture complicated by MRSA infection that led to septic non-union. We treated the septic non union with the technique of induced membrane formation (Masquelet technique). A 33 year old male presented to the outpatient clinic, 2 months after internal fixation of a Gustillo I fracture of the left forearm. There was pus discharge from the operative wound and specimen culture was positive for MRSA. Initially the patient received conservative treatment with antibiotics for a period of one month. However, the patient returned with the same clinical presentation. The patient was then treated with hardware removal of the ulna and debridement of the septic non-union. The formed $5 \mathrm{~cm}$ bone defect was filled with cement spacer and the ulnar bone was fixed with external fixation. Eight weeks later, the spacer was removed and the bone gap was filled with autologous cancellous bone graft from iliac crest. Five months after grafting, the patient was reviewed. No clinical or functional problems were noted and osseous consolidation of the ulnar bone was confirmed in plain x-rays. The Masquelet technique is a promising alternative treatment for the management of infected long bone non-unions of the upper extremity.
\end{abstract}

Key words: Septic non-union, bone defect, masquelet technique.

\section{Introduction}

Trauma, pseudoarthrosis, bone infection, bone necrosis, bony tumours and congenital bone diseases are among the commonest causes of bone defects. ${ }^{[1]}$ Reconstructing a bone defect, especially those of a considerable size above $5 \mathrm{~cm}$, is a challenge in orthopaedic society. The methods usually applied are bone transfer (distraction osteogenesis) utilising external fixation osteosynthesis, and free vascularised bone grafting [1]. Graft absorption or graft incorporation failure in defects greater than $5 \mathrm{~cm}$ is a usual complication [2].

Corresponding author: Christos K. Kyriakopoulos, Orthopaedic Surgeon Ph.D., General Hospital of Attica 'KAT', Nikis 2, Kifissia, P.C. 145-61, Athens, Greece, E-mail: c.kyriakopoulos@yahoo.com.
In cases of septic non union the gold standard of management is the removal of infected bone tissue and filling of the formed bone defect [2]. The purpose of this paper is to present a rare case of MRSA septic non-union of the ulna, treated with Masquelet technique.

\section{Case}

A 33 year old male patient, with no significant past medical history, suffered a left forearm fracture of both radius diaphysis (closed) and ulna diaphysis (open Gustillo type-I) (see Fig. 1). The patient was initially treated with internal fixation of both bones. Two months postoperatively, the patient returned to the outpatient's clinic with left forearm pain and pus discharged from the ulnar surgical wound (see Fig. 2). 


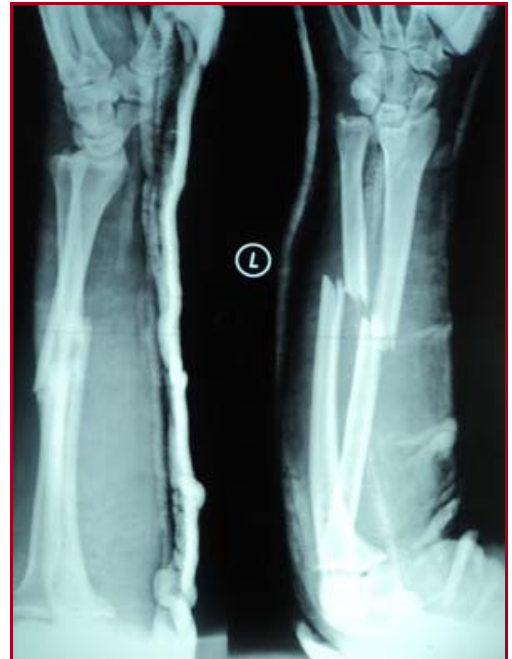

Fig. 1 Preoperative radiograph showing mid-shaft fracture of left forearm.

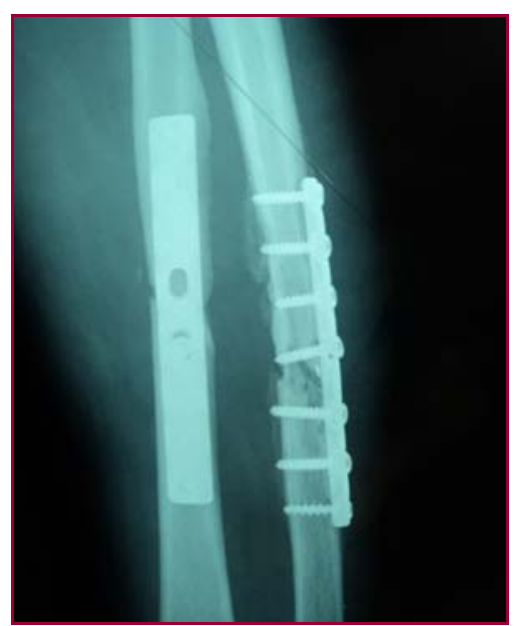

Fig. 2 Radiograph 8 weeks after primary internal fixation.

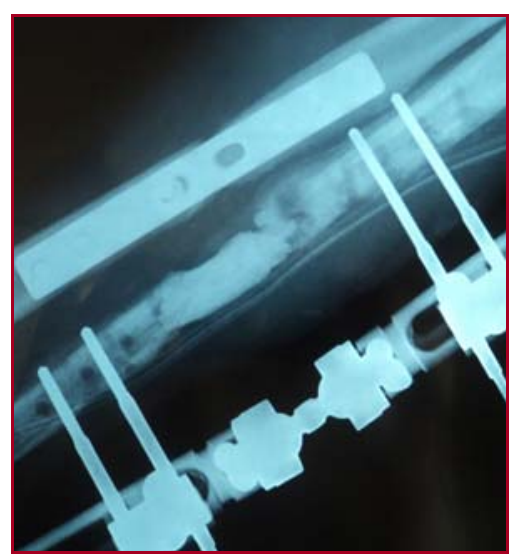

Fig. 3 Intra-operative radiograph showing bone cement as a spacer in mid-shaft of ulna with external fixator. The ulnar fracture was reduced in its proper anatomic position with one-sided external fixation and bone cement imbued with Tobramycin and Vancomycin.
On examination the involved area was tender and swollen. The patient was afebrile with inflammatory markers mildly deranged (CRP 25 mg/dl, ESR 42 $\mathrm{mm} / \mathrm{h}$ ). Wound cultures were positive for MRSA sensitive to Teicoplanin and Rifampicin. The patient received the proper antibiotics i.v. for one month. At the end of that period, all laboratory tests were negative, the wound was healed and the patient was asymptomatic. He was then advised to continue treatment with oral antibiotics (Cloxacillin sodium and Rifampicin). However, one month later the patient revealed again symptoms of discharge and local infection. Surgical treatment was then decided.

\subsection{Surgical Technique}

Primary osteosynthesis implants of the ulna were removed and debridement of the infected and necrotic bone segments was performed. The resulting bone defect was $5 \mathrm{~cm}$ and initially treated with one-sided external fixation. The defect was filled with bone cement with Tobramycin and Vancomycin (see Fig. 3). Soft tissue debridement was also performed. The radius fracture was healed and no further treatment was needed. The patient received postoperatively i.v. Teicoplanin and Rifampicin for 4 weeks. By the end of the $8^{\text {th }}$ postoperative week, the cement spacer was removed and the bone defect was filled with autologous cancellous bone graft from the iliac crest (see Fig. 4). Special care was taken to keep the reactive tissue membrane surrounding the cement intact. The void was thoroughly cleaned with normal saline irrigation to remove any residual debris. There were no macroscopic signs of infection or necrotic tissue recognised into the void and morcellized autologous iliac crest cancellous bone mixed with bone marrow was finally placed into the void. The membrane was approximated with interrupted absorbable sutures. An ulnar splint was placed which remained on site for 4 weeks. The patient followed a special physiotherapy protocol. Regular clinical and radiological follow-up was scheduled for 18 months 


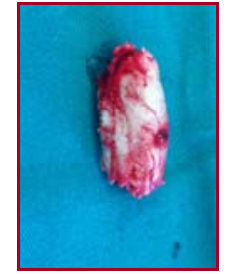

(a)

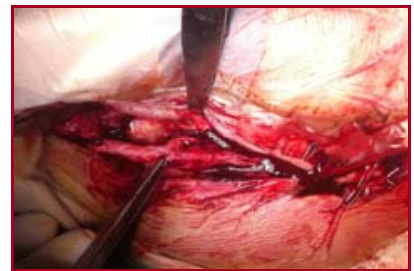

(b)

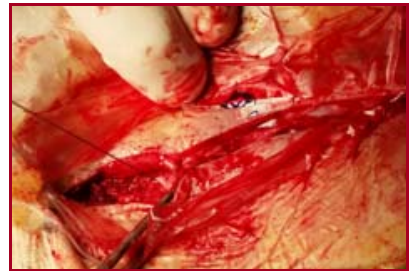

(c)

Fig. 4 At the end of the $8^{\text {th }}$ postoperative week, the bone spacer was removed. a. Photograph showing bone cement after removing, b. Intra-operative photograph showing the cavity and the membrane induced after cement removal c. The membrane was approximated with interrupted sutures of absorbable material and subsequent layers closed.

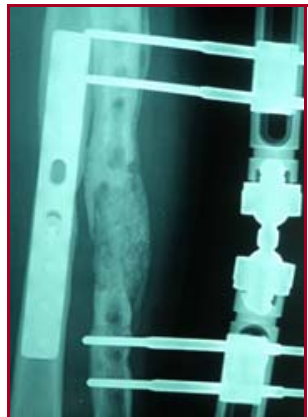

(a)

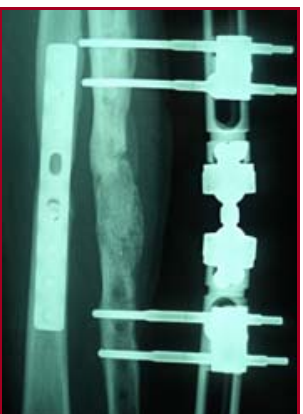

(b)

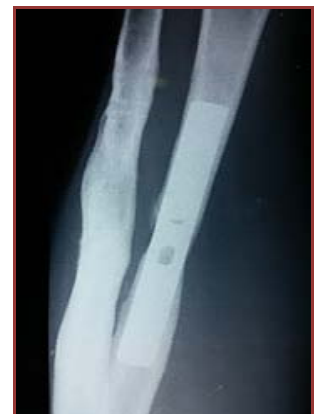

(c)

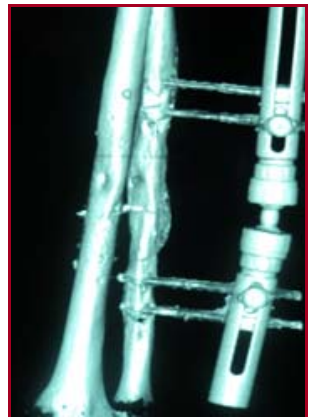

(d)

Fig. 5 Radiographs after a. 6 weeks, b. 3 months, c. 18 months and d. CT-Tomography after 5 months from the second stage of the 'Masquelet' technique.

(see Fig. 5). At the end of the $5^{\text {th }}$ month, the patient did not complain of any clinical or functional problems. Six moths postoperatively, osseous consolidation was confirmed with CT scan and one month later the external fixator was removed. Recently, 18 months after the operation, the patient is free of symptoms.

\section{Discussion}

Managing large $(>5 \mathrm{~cm}$ ) bone defects, especially those with a septic background is a treatment challenge for the orthopaedic surgeon. The prevalent methods employed are osseous distraction and the placement of vascularised bone grafts, usually of peroneal or iliac origin. The drawbacks of the first method are infection around the fixator pins, inflammatory infiltration of the neighbouring joint space, a relatively long time of rehabilitation, high cost and a considerable non-union rate. The second method stipulates extensive operative experience, specialised medical staff (microsurgical techniques) and runs the risk of necrosis or resorption of the graft, increased morbidity of the donor site and extended operative times [2, 3].

The Masquelet technique is an alternative method for bone defect management, comprised of two stages, each with its own "tips and tricks", in order to reduce relevant complications and increase effectiveness [3]. The first stage, known as "formation of the induction membrane" includes bone debridement and filling of the gap with a cement spacer (PMMA). In the second stage, which takes place usually 8 weeks later known as "cancellous bone grafting”, the spacer is removed and replaced with cancellous bone [1]. The technique was first described by the French surgeon Masquelet in 1986 in his attempt to treat bone defects sizing more than $15 \mathrm{~cm}[4,5]$.

The PMMA (Polymethyl Methacrylate) spacer used in the method serves a two-fold purpose. The first is a mechanical one; it gives structural support and simultaneously prevents the filling of the defect with soft tissue. The second is a biological one; it promotes the formation of the cement-surrounding membrane. Histological studies on rabbits demonstrated extensive 
neovascularisation in the bone defect, whereas immunohistochemical studies denoted production of growth hormones (VEGF,TGF-b1) in a time period of two weeks, as well as the osteoinductive growth hormone BMP-2 (bone morphogenetic protein 2), with peak output at 9 weeks [3, 5-10]. These molecules are vital to bone formation, metabolism and remodelling [6]. The interior part of this "pseudomembrane" imitates epithelium and the exterior part includes fibroblasts, myoblasts and collagen [4]. This membrane has the distinct advantage of contributing to the excitation of marrow cell proliferation and differentiation to the osteoblast line, while withholding bone resorption. Also, it possesses a rich network of capillaries (neovascularisation), promotes corticalisation of cancellous bone, and has osteoinductive action and moderate osteogenic properties $[6,9,11]$.

It is well known in the orthopaedic society that microbial infections on metallic implants and prostheses are one of the most prominent causes of morbidity and implant failure. Antimicrobial therapy to these infections becomes even more challenging when the pathogen is staphylococcus aureus and especially the MRSA (methicillin resistant strain). In the reported case the wound cultures taken intraoperatively were positive for MRSA. Based on the sensitivities provided by the laboratory cultures, the combination of Teicoplanin and Rifampicin was chosen as an appropriate regimen. This has been widely published and proved to be one of the most effective combinations to treat such infections [12]. However, despite the noticeable clinical improvement and restoration of inflammatory markers to normal ranges within a month, the infection recurred both biochemically and clinically. Also, based on sensitivities, the cement spacer was mixed with Tobramycin and Vancomycin as a frontline defence against MRSA and a safeguard for the method applied. The external fixator remained in situ for 6 weeks after the bone grafting, until safe results were obtained from both clinical and radiological standpoints.

\section{Conclusion}

In conclusion, the Masquelet method in cases of septic non-union of the upper limb with concurrent bone defect sizing more than $5 \mathrm{~cm}$, in combination with antibiotic-mixed cement and use of external fixation, is an alternative, safe, low cost and effective therapeutic option.

\section{References}

[1] Motsitsi, N. S. 2012. "Masquelet's Technique for Management of Long Bone Defects: From Experiment to Clinical Application.” East and Central African Journal of Surgery 17 (2).

[2] Shameem, A., and Mukesh, K. 2013. “Mariappan Selvamari. Induced Membrane Formation in a Case of Infected Gap Nonunion of Radius: Case Report.” J. Clin. Orthop. Trauma 4 (3): 147-50.

[3] Giannoudis, P. V., Faour, O. T. G., Kanakaris, N., and Dimitriou, R. 2011. "Masquelet Technique for the Treatment of Bone Defects: Tips-tricks and Future Directions.” Injury 42 (6): 591-8.

[4] Masquelet, A. C., and Obert, L. 2010. "Induced Membrane Technique for Bone Defects in Hand and Wrist.” Chir Main 29 (suppl 1): S221-S224.

[5] Pelissier, P., Boireau, P., Martin, D. et al. 2003. "Bone Reconstruction of the Lower Extremity: Complications and Outcome.” Plastic and Reconstructive Surgery 111 (7): 2223-9.

[6] Pelissier, Ph., Masquelet, A. C., Bareille, R. et al. 2004. "Induced Membrane Secrete Growth Factors Including Vascular and Osteoconductive Factors and Could Stimulate Bone Regeneration.” Journal of Orthopaedic Research 22: 73-9.

[7] Meinig, R. P. 2010. "Clinical Use of Resorbable Polymeric Membranes in the Treatment of Bone Defects.” Orthop. Clin. N. Am. 41: 39-47.

[8] Pelissier, P., Martin, D., Baudet, J., Lepreux, S., and Masquelet, A. C. 2002. "Behavior of Cancellous Bone Graft Placed in Induced Membranes.” Br. J. Plast Surg. 55: 596-8.

[9] Spinella-Jaegle, S., Roman, S., Faucheu, C., et al. 1998. "Opposite Effects of BMP-2 and TGF Beta-1 on Osteoblast Differentiation.” Bone 29: 323-30.

[10] Assal, M., Stern, R. 2014. "The Masquelet Procedure Gone Awry.” Orthopedics 37 (11): e1045-8.

[11] Woon, C. Y., Chong, K. W., and Wong, M. K. 2010. "Induced Membranes e a Staged Technique of Bone 

of the Ulna-A case Report

Grafting for Segmental Bone Loss: A Report of Two Cases and a Literature Review.” J. Bone Joint Surg. Am. 92: 196-201.

[12] Schaad, H. J., Chuard, C., Vaudaux, P., Waldvogel, F. A., Lew, D. P. 1994. "Teicoplanin Alone or Combined with Rifampin Compared with Vancomycin for Prophylaxis and Treatment of Experimental Foreign Body Infection by Methicillin-Resistant Staphylococcus Aureus." Antimicrobial Agents Chemotherapy 38 (8): 1703-10.

[13] Bayer, A. S., and K. Lam. 1985. "Efficacy of Vancomycin plus Rifampin in Experimental Aortic-Valve Endocarditis due to Methicillin-Resistant Staphylococcus aureus: in vitro-in vivo Correlations.” J. Infect. Dis. 151:
157-65.

[14] Van der Auwera, P., Meunier Carpentier, F. and Klastersky, J. 1983. "Clinical Study of Combination Therapy with Oxacillin and Rifampin for Staphylococcal Infections.” Rev. Infect. Dis. 5 (Suppl.3): S515-S522.

[15] Faville, R. J., Jr., D. E. Zaske, E. L. Kaplan, K. Crossley, L., Sabath, D., and Quie, P. G. 1978. "Staphylococcus aureus Endocarditis. Combined Therapy with Vancomycin and Rifampin.” JAMA 240: 1963-5.

[16] Norden, C. W., and M. Shaffer. 1983. "Treatment of Experimental Chronic Osteomyelitis due to Staphylococcus aureus with Vancomycin and Rifampin.” J. Infect. Dis. 147: 352-7. 